\title{
Retraction Note: SNPs in genes implicated in radiation response are associated with radiotoxicity and evoke roles as predictive and prognostic biomarkers
}

\author{
Ghazi Alsbeih ${ }^{1,4^{*}}$, Medhat El-Sebaie${ }^{2}$, Najla Al-Harbi ${ }^{1}$, Khaled Al-Hadyan ${ }^{1}$, Mohamed Shoukri ${ }^{3}$ and Nasser Al-Rajhi ${ }^{2}$
}

\section{Retraction}

The authors are retracting this article [1] because the data have already been published in [2] making this a redundant publication. Ghazi Alsbeih, Najla Al-Harbi, Khaled Al-Hadyan, Mohamed Shoukri and Nasser Al-Rajhi agree with this retraction. Medhat El-Sebaie did not respond to our correspondence.

\begin{abstract}
Author details
${ }^{1}$ Radiation Biology Section, Biomedical Physics Department, King Faisal Specialist Hospital and Research Centre, P.O. Box 3354, Riyadh 11211, Saudi Arabia. ${ }^{2}$ Radiation Oncology Section, Oncology Centre, King Faisal Specialist Hospital and Research Centre, P.O. Box 3354, Riyadh 11211, Saudi Arabia. ${ }^{3}$ National Biotechnology Center, King Faisal Specialist Hospital and Research Centre, P.O. Box 3354, Riyadh 11211, Saudi Arabia. ${ }^{4}$ Radiation Biology Section, Biomedical Physics Department, KFSHRC, MBC-03, P.O. Box 3354, Riyadh 11211, Saudi Arabia.
\end{abstract}

Received: 11 April 2018 Accepted: 13 April 2018 Published online: 26 April 2018

\section{References \\ 1. Alsbeih G, El-Sebaie M, Al-Harbi N, Al-Hadyan K, Shoukri M, Al-Rajhi N. SNPS in genes implicated in radiation response are associated with radiotoxicity and evoke roles as predictive and prognostic biomarkers. Radiat Oncol. 2013;8:125. https://doi.org/10.1186/1748-717X-8-125. \\ 2. Alsbeih G, El-Sebaie M, Al-Rajhi N, Al-Harbi N, Al-Hadyan K, Al-Qahtani S, Alsubael M, Al-Shabanah M, Moftah B. Among 45 variants in 11 genes, HDM2 promoter polymorphisms emerge as new candidate biomarker associated with radiation toxicity. 3Biotech. 2014;4:137-48 (First Online 26 April 2013)}

\footnotetext{
* Correspondence: galsbeih@kfshrc.edu.sa

${ }^{1}$ Radiation Biology Section, Biomedical Physics Department, King Faisal

Specialist Hospital and Research Centre, P.O. Box 3354, Riyadh 11211, Saudi

Arabia

${ }^{4}$ Radiation Biology Section, Biomedical Physics Department, KFSHRC, MBC-03,

P.O. Box 3354, Riyadh 11211, Saudi Arabia
} 